\title{
Local and remote control measures for Networked Control Systems
}

\author{
Tilemachos Matiakis, Sandra Hirche and Martin Buss
}

\begin{abstract}
In this article we propose a novel approach for networked control systems with unknown constant time delay. A distributed controller is considered, under the assumption that static controllers directly connected to the plant, can be implemented. Stability and sensitivity to time delay goals can be conjointly defined. The necessary and sufficient conditions for the existence of a controller which guarantees delayindependent stability and a frequency dependent maximum of the norm of the sensitivity function with respect to time delay are formulated as a feasibility problem with polynomial matrix inequality constraints. In a numerical example we show that the proposed formulation can give usefull solutions in non-trivial cases.
\end{abstract}

\section{INTRODUCTION}

$\mathbf{I}$ $\mathrm{N}$ networked control systems (NCS) the plant and the controller are spatially separated, and connected through a communication network, see [1]-[3] for an overview. The motivation for replacing classical point-to-point architecture with NCS originates, among others, from the flexible reconfiguration NCS offer ; nodes can be added or removed without additional wiring effort. The number of active nodes sharing the communication line has an effect on the communication time delay, the packet loss, and the available communication bandwidth, parameters which are therefore not exactly known during the controller design. Advantageously, NCS offer additional degrees of freedom for the controller design, i.e. some limited computational power is available on the plant side, which can be used to implement low order controllers.

In this article the unknown constant time delay challenge is addressed. Time delay in the control loop deteriorates the performance and may lead to instability. For a general overview on constant time delay systems see [4], [5]. Constant time delay methods are distinguished between delaydependent and delay-independent, according to whether a bound on the time delay value is necessary for stability or not. Delay-independent methods are usually based on the small gain theorem, i.e. the gain of the open loop transfer function must be smaller than one over all frequencies, which is known to be rather conservative.

Here, a novel distributed controller approach is proposed for delay-independent stability, under the assumption that only static controllers can be implemented on the plant side. A two degrees of freedom controller is considered. The first

This work was supported in part by the German Research Foundation (DFG) within the Priority Programme SPP 1305 "Regelungstheorie digital vernetzter dynamischer Systeme".

All authors are with the Institute of Automatic Control Engineering Technische Universität München D-80290 München, Germany www.lsr.ei.tum.de, T.Matiakisetum.de, hirchedtum. de, M.Bussdieee.org controller part is designed for zero time delay, while the second guarantees delay-independent stability and low sensitivity to time delay. Low sensitivity to time delay ensures that the performance gracefully degrades with increasing time delay value. Combined with good performance for zero time delay, satisfactory performance is achieved in a wide range of time delay values.

Delay-independent stability as well as a frequency dependent maximum of the norm of the sensitivity function with respect to time delay can be guaranteed by the $H_{\infty}$ norm of the loop, where the time delay and the "extended by the static controller plant" reside. The necessary and sufficient conditions for the existence of a controller which guarantees a maximum $H_{\infty}$ norm of the above mentioned loop are formulated as a feasibility problem with polynomial matrix constraints. In a numerical example it is shown that the proposed approach can supply useful solutions in nontrivial cases.

This work is a continuation of the results in [6], [7] and was originally inspired by the scattering transformation [8], [9]. The scattering transformation, is a method frequently encountered in force feedback telepresence systems with arbitrary constant time delay. Major contribution of this article compared to the above is the formulation of the controller design problem as a feasibility problem with polynomial matrix constraints, as well as the consideration of a more general transformation.

The remainder of this article is organized as follows. Section II presents the necessary background and Section III the problem formulation. The stability analysis is given in Section IV and performance issues are discussed in Section V. In Section VI a controller design strategy is presented and in Section VII a numerical example in given. Conclusions are presented in Section VIII.

\section{PRELIMINARIES}

Notation : The set of non-negative real numbers is denoted by $\mathbb{R}_{+}$, the Euclidean space of dimension $m$ by $\mathbb{R}^{m}$, and the set of real and complex valued matrices with dimensions $n \times m$ by $\mathbb{R}^{n \times m}$ and $\mathbb{C}^{n \times m}$ respectively. For a matrix $M$ the transposition and conjugate transposition are denoted by $M^{T}$ and $M^{*}$. The notation $M>0$ is used to denote its positive definiteness $(" \geq ", "<$ ", $\leq$ " for positive semi-definiteness, negative definiteness, negative semidefiniteness respectively). With $I$, the unit matrix is denoted and with $\operatorname{diag}\left[M_{1}, \ldots, M_{n}\right]$, the block diagonal matrix with diagonal elements $M_{1}$ until $M_{n}$.

We consider linear-time-invariant (LTI), controllable and observable systems given by 


$$
\Sigma: \dot{x}=A x+B u, \quad y=C x+D u
$$

where $x(t) \in \mathbb{R}^{n}, u(t) \in \mathbb{R}^{m}, y(t) \in \mathbb{R}^{p}$ are the state, input and output vectors respectively. With capital letters, the Laplace transforms of the corresponding signals are denoted and with $G(s)=C(s I-A)^{-1} B+D$ the transfer function of (1), where $s=\sigma+j \omega$ is the Laplace variable. The norm of a transfer function at $\omega$ is denoted by $|G(j \omega)|$ and its $H_{\infty}$ norm, i.e. $\max |G(j \omega)|, \forall \omega$, by $\|G\|_{\infty}$. $L_{2 e}$ denotes the extended space of Lebesgue integrable functions. For convenient notation, when non-ambiguous the time argument $t$ and the Laplace variable $s$ are dropped.

\section{A. Sensitivity function}

The sensitivity function of a system (1) with transfer function $G$, with respect to a parameter $T$, is given by

$$
S_{T}^{G}(s)=\frac{d G(s) / G(s)}{d T / T}=\frac{T}{G(s)} \frac{d G(s)}{d T} .
$$

Low $\left|S_{T}^{G}(s)\right|$ implies that a variation in $T$ slightly affects $G$, i.e. the input-output behaviour of the system.

\section{B. The Kalman-Yakubovich-Popov lemma}

Given a controllable and observable system (1) with transfer function $G(s)$ and the matrices $Q=Q^{T} \in \mathbb{R}^{m \times m}, S \in$ $\mathbb{R}^{p \times m}, R=R^{T} \in \mathbb{R}^{p \times p}$, the following statements are equivalent [10] :

1) For all $\omega \in \mathbb{R}$ with $\operatorname{det}(j \omega I-A) \neq 0$

$$
\left[\begin{array}{c}
I \\
G(j \omega)
\end{array}\right]^{*}\left[\begin{array}{cc}
Q & S \\
S^{T} & R
\end{array}\right]\left[\begin{array}{c}
I \\
G(j \omega)
\end{array}\right] \leq 0
$$

2) There exists a symmetric matrix $P=P^{T}$ such that

$$
\left[\begin{array}{rr}
A^{T} P+P A & P B \\
B^{T} P & 0
\end{array}\right]+\left[\begin{array}{cc}
0 & I \\
C & D
\end{array}\right]^{T}\left[\begin{array}{rr}
Q & S \\
S^{T} & R
\end{array}\right]\left[\begin{array}{cc}
0 & I \\
C & D
\end{array}\right] \leq 0 .
$$

\section{PROBLEM FORMULATION}

The plant $\Sigma_{p}$ is given by (1), with $x_{p} \in \mathbb{R}^{n_{p}}, u_{p} \in \mathbb{R}^{m}, y_{p} \in$ $\mathbb{R}^{p}$ being its state, input and output vectors respectively, see Fig. 1. On the plant side a static-output-feedback-inputfeedforward (SOFIF) controller is used. The local static controller with input and output vectors $\left[\begin{array}{ll}u_{r} & y_{p}\end{array}\right],\left[\begin{array}{ll}u_{p} & v_{r}\end{array}\right] \in \mathbb{R}^{m+p}$ respectively, is described by an invertible transformation $M \in \mathbb{R}^{(m+p) \times(m+p)}$ between the plant input-output vector $z_{p}$, and the right hand transmitted through the network variables $s_{r}$, i.e.

$$
s_{r}=M z_{p}, \text { with } z_{p}^{T}=\left[\begin{array}{ll}
u_{p} & y_{p}
\end{array}\right], \quad s_{r}^{T}=\left[\begin{array}{ll}
u_{r} & v_{r}
\end{array}\right] .
$$

The remote controller is decomposed in the same structure, i.e. a static part described by the inverse transformation $M^{-1}$ between the left hand transmitted through the network variables $s_{l}$ and the input-output vector $z_{c}$, i.e.

$$
z_{c}=M^{-1} s_{l}, \text { with } z_{c}^{T}=\left[\begin{array}{ll}
y_{c} & u_{c}
\end{array}\right] \quad s_{l}^{T}=\left[\begin{array}{ll}
u_{l} & v_{l}
\end{array}\right]
$$

and the dynamic controller $\Sigma_{c}$ given by (1), with $x_{c} \in \mathbb{R}^{n_{c}}, e \in \mathbb{R}^{p}, y_{c} \in \mathbb{R}^{m} \quad$ being its state, input and output vectors respectively, see Fig. 1. By $e=w-u_{c}$ the control error is denoted, with $w \in L_{2 e}$ being the desired value and $u_{c}$ the lefthand output of $M^{-1}$. In order to avoid confusion, in the following we refer to the local and remote SOFIF controllers simply as transformation $M$.

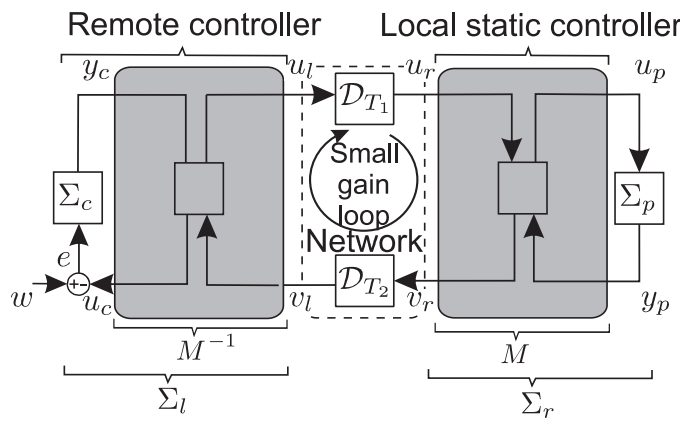

Fig. 1. Networked control system with local and remote control measures.

The communication network is modelled as a forward time delay operator $\mathscr{D}_{T_{1}}$ (controller to plant channel) and a backward time delay operator $\mathscr{D}_{T_{2}}$ (plant to controller channel) with inputs $u_{l}(t), u_{r}(t)$ and outputs $v_{r}(t), v_{l}(t)$, see Fig. 1. The relation between the left and right transmitted variables is given by $u_{r}(t)=u_{l}\left(t-T_{1}\right)$ and $v_{l}(t)=v_{r}\left(t-T_{2}\right)$. The time delays $T_{1}, T_{2} \in \mathbb{R}^{+}$are constant but unknown. For further reference, the following subsystems are defined: $\quad \Sigma_{r}: u_{r}(\cdot) \rightarrow v_{r}(\cdot), \quad \Sigma_{l}: v_{l}(\cdot) \rightarrow u_{l}(\cdot), \Sigma_{O L}=\Sigma_{r} \circ \Sigma_{l}$, see also Fig. 1. Note that the index ()$_{r}$ denotes the right subsystem, including the local controller and the plant, and the index ()$_{l}$ the left, comprising the whole remote controller.

With the proposed controller structure, for zero time delay, when $s_{l}=s_{r}$ (2) (3) holds, we have $z_{c}=M^{-1} s_{l}=M^{-1} s_{r}=M^{-1} M z_{p}=z_{p}$. The left static transformation $M^{-1}$ cancels the right one $M$ and the standard feedback interconnection between the plant $\Sigma_{p}$ and the controller $\Sigma_{c}$ is recovered. In consequence, for zero time delay, $\Sigma_{c}$ can be designed with typical controller design techniques. Note, that the standard approach independently of the time delay is recovered for $M=I$.

In the remainder of this article we consider that the controller $\Sigma_{c}$ is a-priori designed for zero time delay. We furthermore assume, that the closed loop system is well posed, i.e. for each input signal $w \in L_{2 e}$ there exists a unique solution for the signals $e, u_{c}, y_{c}, u_{l}, v_{l}, u_{r}, v_{r}, u_{p}, y_{p}$ that causally depends on $w$. Clearly, considering the standard approach, i.e. $M=I$, the closed loop system can be unstable, as shown e.g. in [11] for passive subsystems. The main result of this paper are the necessary and sufficient conditions of the existence of a transformation $M$ which guarantees delay-independent stability as well as a frequency dependent maximum for the norm of the sensitivity function of the closed loop system with respect to time delay.

\section{STABILITY ANALYSIS}

In the rest of this article we restrict to single-inputsingle-output case, i.e. $m=1$. The elements of the matrix $M \in \mathbb{R}^{2 \times 2}$ are denoted by $m_{11}=a, m_{12}=b, m_{21}=c, m_{22}=d$, where we choose $a, b, c, d$ such that $\operatorname{det} M \neq 0$. The closed loop transfer function $G(s)$ from the reference input $W$ to the plant output $Y_{p}$ is computed by the transformation equations (2),(3) to be, 


$$
\begin{gathered}
G(s)=G_{0}(s) G_{t r}(s) e^{-s T_{1}}, \text { where } G_{0}(s)=\frac{G_{p}(s) G_{c}(s)}{1+G_{p}(s) G_{c}(s)}, \\
G_{t r}(s)=\frac{1-G_{O L}(s)}{1-G_{O L}(s) e^{-s T}}, \text { and } G_{O L}=G_{r} G_{l} \\
\text { with } \quad G_{r}=\frac{V_{r}}{U_{r}}=\frac{c+d G_{p}}{a+b G_{p}}, \quad G_{l}=\frac{U_{l}}{V_{l}}=\frac{b-a G_{c}}{d-c G_{c}}
\end{gathered}
$$

According to (4) the system can be seen as a series connection of the standard closed loop system without time delay $G_{0}$, and the system $G_{t r}$ which describes the influence of the time delay and the transformation $M$ in the closed loop behaviour. Under this setup delay-independent stability is equivalent to a small gain condition of the loop where the time delay and the "extended by the local static controller plant" reside, see Fig. 1

Proposition 1: The closed loop system is delayindependently stable if and only if $G_{O L}$ is stable and

$$
\left|G_{O L}\right|<1 \text {, for all } \omega>0 \text {. }
$$

Proof: For delay independent stability the system must be stable for $T_{1}=T_{2}=\infty$, i.e. when there is no connection between plant and controller. This means that $G_{r}, G_{l}$ and consequently $G_{O L}$ must be stable. Now, consider the full open loop transfer function, including the forward and backward time delay $T=T_{1}+T_{2}$, i.e. $G_{O L} e^{-j \omega T}$. For stability $\left|G_{O L} e^{-j \omega T}\right|<1$ must be satisfied, when $\arg \left\{G_{O L} e^{-j \omega T}\right\} \leq$ $-180^{\circ}$ holds. For arbitrary $T$ and $\omega \neq 0, e^{-j \omega T}$ defines an arbitrary phase shift. Thus, for all $\omega>0,\left|G_{O L}\right|<1$ must hold.

The addition of the transformation $M$ allows a larger class of controllers for delay-independent stability compared to the standard small gain theorem. This may lead to less conservative controller design. In fact, even delay-dependent approaches are outperformed as shown in [6] for a special case of the proposed approach.

Remark 1: Instead of the necessary and sufficient stability condition (7), the condition $\left\|G_{O L}\right\|_{\infty}<1$ can be used, as the computation of $\left\|G_{O L}\right\|_{\infty}$ is amenable to many analysis tools, e.g. linear matrix inequalities. Conservatism of the stability condition $\left\|G_{O L}\right\|_{\infty}<1$ comes from the fact that $\left|G_{O L}\right|$ must be less than one in the zero frequency as well.

Remark 2: Proposition 1 implies strict stability of $\Sigma_{r}, \Sigma_{l}$. $\Sigma_{r}$ is the combination of the plant $\Sigma_{p}$ with the local SOFIF controller. Thus, in case of an unstable plant, it must be prestabilized by the static output-feedback, restricting the approach to static-output-feedback stabilizable plants. If that's not the case, a higher order dynamic transformation $M$ should be considered. This is subject for future research.

Remark 3: Special cases of the proposed setup are the scattering transformation used in passive telepresence systems with time delay and packet loss in the communication network [11], as well as the more general transformation introduced by the authors in [7] for inputfeedforward-output-feedback passive systems. In fact, in all these cases, instead of the stability condition (7), the more conservative one $\left\|G_{r}\right\|_{\infty}\left\|G_{l}\right\|_{\infty}<1$ is considered as $\left\|G_{O L}\right\|_{\infty} \leq\left\|G_{r}\right\|_{\infty}\left\|G_{l}\right\|_{\infty}$. By resolving to this more conservative condition many scattering based approaches for timevarying delay [12] and packet loss [13] are straightforward to apply here. However, here, we concentrate on the necessary and sufficient conditions for constant time delay.

\section{PERFORMANCE ANALYSIS}

In the following, the sensitivity to time delay and the steady state behaviour are discussed.

\section{A. Sensitivity to time delay}

According to the previous section in the remainder of this section it is assumed that $\left\|G_{O L}\right\|_{\infty}<1$, i.e. the system is delay independently stable. Here, it is shown that a lower bound on $\left\|G_{O L}\right\|_{\infty}<1$ can further guarantee a frequency dependent maximum of the norm of the sensitivity function with respect to time delay. The sensitivity function of the closed loop system with respect to the round trip time delay $T=T_{1}+T_{2}$ is given by the infinite dimensional transfer function

$$
S_{T}^{G^{*}}=\frac{T}{G^{*}} \frac{d G^{*}}{d T}=s T e^{-s T} \frac{G_{O L}}{1-G_{O L} e^{-s T}},
$$

where $G^{*}(s)=G_{0}(s) G_{t r}(s)$ is the transfer function (4) without the purely time shifting part $e^{-s T_{1}}$. For $S_{T}^{G^{*}}$ the next Theorem holds

Theorem 1: When $\left\|G_{O L}\right\|_{\infty}<1$ for each $\omega_{0}$ holds

$$
\left|S_{T}^{G^{*}}\left(j \omega_{0}\right)\right| \leq \frac{\omega_{0} T\left\|G_{O L}\right\|_{\infty}}{1-\left\|G_{O L}\right\|_{\infty}} .
$$

Proof: For the norm of the sensitivity function we have

$\left|S_{T}^{G^{*}}\left(j \omega_{0}\right)\right|=\frac{\omega_{0} T\left|G_{O L}\right|}{\mid 1-G_{O L} e^{-j \omega_{0} T \mid}} \leq \frac{\omega_{0} T\left|G_{O L}\right|}{1-\left|G_{O L}\right|} \leq \frac{\omega_{0} T\left\|G_{O L}\right\|_{\infty}}{1-\left\|G_{O L}\right\|_{\infty}}$ where the dependence on $j \omega_{0}$ is suppressed for convenience of notation.

From the derivative of (8) with respect to $\left\|G_{O L}\right\|_{\infty}$ it is straightforward to see that the right part of (8) is a strictly increasing function of $\left\|G_{O L}\right\|_{\infty}$. Thus, instead of minimizing the norm of the infinite dimensional function $S_{T}^{G^{*}}$ we can minimize $\left\|G_{O L}\right\|_{\infty}$. Note that this conforms to the small gain stability requirement $\left\|G_{O L}\right\|_{\infty}<1$. However, Theorem 1 guarantees a worst case bound for the sensitivity function and not the actual value.

Insensitivity, i.e. $S_{T}^{G^{*}}=0$, can be achieved with a proportional controller $G_{c}(s)=\frac{b}{a}$, independently of the plant. This follows from substituting $G_{c}$ in (5) resulting in $G_{O L}=0 \Rightarrow S_{T}^{G^{*}}=0 \Rightarrow G_{t r}(s)=1$. The closed loop transfer function (4) reduces to $G(s)=G_{0}(s) e^{-s T_{1}}$ with the time shifting part having no effect on the transient response. This is the limit case in which the controller is completely locally implemented. Only the reference input $w$ is transmitted through the communication network, as implied by $G_{O L}=0$. In general, a proportional controller does not meet the performance requirements and a compromise should be made between performance and sensitivity to time delay.

\section{B. Steady state behaviour}

The steady state behaviour of the proposed controller structure is equivalent to the steady state behaviour of the typical feedback interconnection of plant and controller without time delay, as easily derivable by setting $s=0$ in (4) and (5) resulting in $G(0)=G_{0}(0)$. In terms of steady state error the proposed approach clearly outperforms the standard small gain approach which is the 
only alternative delay-independent method without local control measures. The standard small gain approach requires $\left|G_{c}(j \omega) G_{p}(j \omega)\right|<1, \omega>0$, i.e. free integrators in the open loop are not allowed. In the proposed approach free integrators in plant or controller do not necessarily appear as free integrators in $G_{O L}$ (5). As a result delayindependent stability can still be guaranteed by Proposition 1 while the integrator guarantees steady state error zero. This can be easily demonstrated using examples, e.g. $G_{p}(s)=$ $\frac{1}{s+1}, G_{c}(s)=\frac{s+1}{s(s+10)}, a=0.866, b=0.5, c=-0.5, d=0.866$.

\section{Controller Design}

Based on the above, the problem of guaranteeing delayindependent stability as well as a frequency dependent maximum for the norm of the sensitivity function with respect to time delay, can be expressed as a minimization problem of $\left\|G_{O L}\right\|_{\infty}$. In the following it is shown that the controller design problem of finding a transformation $M$ so that $\left\|G_{O L}\right\|_{\infty}$ is bounded by a specific value can be expressed as a feasibility problem with polynomial matrix constraints. In the following theorem the optimization parameters are denoted by bold letters.

Theorem 2: $\left\|G_{O L}\right\|_{\infty} \leq \gamma_{e x}$ if and only if there are $\gamma_{\mathbf{c}}, \gamma_{\mathbf{p}} \in \mathbb{R}^{+}, \mathbf{l}_{\mathbf{1}}, \mathbf{l}_{\mathbf{2}}, \mathbf{l}_{\mathbf{3}}, \mathbf{l}_{\mathbf{4}} \in \mathbb{R}$, and symmetric matrices $\mathbf{P}_{\mathbf{p}}, \mathbf{P}_{\mathbf{c}}, \mathbf{P}_{\mathbf{e x}}$ so that for $i \in\{e x, p, c\}$,

$$
\begin{gathered}
F_{i}=\left[\begin{array}{rr}
A_{i}^{T} \mathbf{P}_{\mathbf{i}}+\mathbf{P}_{\mathbf{i}} A_{i} & \mathbf{P}_{\mathbf{i}} B_{i} \\
B_{i}^{T} \mathbf{P}_{\mathbf{i}} & 0
\end{array}\right]+ \\
{\left[\begin{array}{rr}
0 & I \\
C_{i} & D_{i}
\end{array}\right]^{T} \mathbf{Q}_{\mathbf{i}}\left[\begin{array}{rr}
0 & I \\
C_{i} & D_{i}
\end{array}\right] \leq 0,} \\
\mathbf{P}_{\mathbf{p}}>0, \quad \mathbf{P}_{\mathbf{c}}>0, \quad \mathbf{l}_{\mathbf{1}} \mathbf{l}_{\mathbf{4}}=\mathbf{l}_{\mathbf{2}} \mathbf{l}_{\mathbf{3}}, \quad \mathbf{l}_{\mathbf{1}} \neq \mathbf{l}_{\mathbf{4}},
\end{gathered}
$$

with $Q_{i}$ for $i \in\{e x, p, c\}$ given by $\mathbf{Q}_{\mathbf{i}}=\mathbf{T}_{\mathbf{i}}^{T} \operatorname{diag}\left\{1,-\gamma_{\mathbf{i}}^{2}\right\} \mathbf{T}_{\mathbf{i}}$,

$$
\begin{gathered}
\mathbf{T}_{\mathbf{e x}}=\left[\begin{array}{llll}
\mathbf{l}_{1} & \mathbf{l}_{2} & -\mathbf{l}_{3} & -\mathbf{l}_{\mathbf{4}} \\
\mathbf{l}_{\mathbf{4}} & \mathbf{l}_{3} & -\mathbf{l}_{2} & -\mathbf{l}_{1}
\end{array}\right], \\
\mathbf{T}_{\mathbf{p}}=\left[\begin{array}{rr}
0 & \mathbf{l}_{3} \\
\mathbf{l}_{3} & \mathbf{l}_{1}
\end{array}\right], \quad \mathbf{T}_{\mathbf{c}}=\left[\begin{array}{rr}
0 & \mathbf{l}_{\mathbf{4}} \\
\mathbf{l}_{4} & -\mathbf{l}_{1}
\end{array}\right],
\end{gathered}
$$

and $A_{e x}, B_{e x}, C_{e x}, D_{e x}$ representing the extended system of the parallel connection of $\Sigma_{p}, \Sigma_{c}$ and $\Sigma_{p c}=\Sigma_{p} \circ \Sigma_{c}$, i.e.

$$
\begin{array}{ll}
A_{e x}=\operatorname{diag}\left[A_{p}, A_{c}, A_{p c}\right] & B_{e x}^{T}=\left[\begin{array}{lll}
B_{p} & B_{c} & B_{p c}
\end{array}\right] \\
C_{e x}=\operatorname{diag}\left[\begin{array}{lll}
C_{p} & C_{c} & C_{p c}
\end{array}\right] & D_{e x}^{T}=\left[\begin{array}{lll}
D_{p} & D_{c} & D_{c p}
\end{array}\right],
\end{array}
$$

and

$$
\begin{aligned}
A_{p c} & =\left[\begin{array}{rr}
A_{p} & 0 \\
B_{c} C_{p} & A_{c}
\end{array}\right] \quad B_{p c}=\left[\begin{array}{r}
B_{p} \\
B_{c} D_{p}
\end{array}\right] \\
C_{p c} & =\left[D_{c} C_{p} C_{c}\right] \quad D_{p c}=D_{c} D_{p} .
\end{aligned}
$$

For a feasible solution $l_{1, f}, l_{2, f}, l_{3, f}, l_{4, f}, d$ can be freely chosen. The rest of the elements of $M$ are given by $a=$ $l_{4, f} / d, b=l_{2, f} / d, c=l_{1, f} d / l_{2, f}$.

Proof : See the appendix.

The boundness of $\left\|G_{O L}\right\|_{\infty}$ by $\gamma_{e x}$ is equivalent to strict stability of $\Sigma_{r}, \Sigma_{l}$, and $\left|G_{O L}(j \omega)\right| \leq \gamma_{e x}, \forall \omega$. It is shown in the proof that a bound of $\left|G_{O L}(j \omega)\right|, \forall \omega$ can be concluded by the values on the $j \omega$ axis of the extended system $\Sigma_{e x}(11)$. The KYP lemma is then used in order to reformulate the condition for the values of $\left|G_{e x}(j \omega)\right|$ to a finite dimensional matrix inequality, i.e. $F_{e x} \leq 0$ (9), based on the state space representation of $\Sigma_{e x}$ (11). Inequalities $F_{p} \leq 0, F_{c} \leq 0$ (9), imply finite gain $L_{2}$ stability $\Sigma_{r}$ and $\Sigma_{l}$ with arbitrary large gains $\gamma_{\mathbf{p}}, \gamma_{\mathbf{c}} \in \mathbb{R}^{+}$respectively, which is equivalent to strict stability.

Remark 4: The optimization parameters $\gamma_{p}, \gamma_{c}$, i.e. the arbitrary large $L_{2}$ gains of $\Sigma_{r}, \Sigma_{l}$, are in real problems bounded by the computational capabilities of the solvers. Therefore, they can be a-priori set to a large constant value, reducing the constraints $F_{p}, F_{c} \leq 0$ (9) from polynomial to quadratic.

Remark 5: Inequalities $F_{p}, F_{c} \leq 0$ (9), which guarantee strict stability of $\Sigma_{r}, \Sigma_{l}$, can be replaced by a number of quadratic inequalities. The stability area for the plant feedback gain in $\Sigma_{r}$ can be a-priori defined based on classical methods, like the Nyquist plot or the root locus. It is shown in the proof that the plant feedback gain in $\Sigma_{r}$ is $-\mathbf{l}_{\mathbf{1}} / \mathbf{l}_{\mathbf{3}}$. In consequence, the constraint for strict stability of $\Sigma_{r}$ can be expressed as a number of inequalities of the form $-\frac{\mathbf{l}_{\mathbf{1}}}{\mathbf{l}_{\mathbf{3}}}<k_{i}$ or

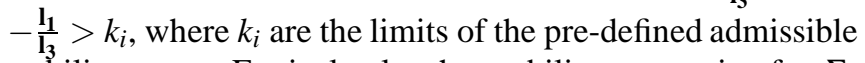
stability areas. Equivalently, the stability constraint for $\Sigma_{l}$ can be expressed by a number of quadratic inequalities of the form $\mathbf{l}_{\mathbf{3}} / \mathbf{l}_{\mathbf{4}}<k_{j}$ or $\mathbf{l}_{\mathbf{3}} / \mathbf{l}_{\mathbf{4}}>k_{j}$.

Remark 6: Any non-convex polynomial constraint can be reformulated into a bilinear one with increased dimension [14]. Bilinear problems are in general hard to solve, and it is known that even the BMI feasibility problem is NPhard [15]. Nevertheless, as shown in the numerical example, the available solvers can give useful solutions in non-trivial cases based on the proposed formulation.

Remark 7: For controller design methods that can be implemented as problems with bilinear constraints, e.g. LQG control, the co-design of $\Sigma_{c}$ and the transformation $M$ is straightforward. The co-design is achieved by considering the controller parameters $A_{c}, B_{c}, C_{c}, D_{c}$ as optimization parameters and adding the additional performance constraints. Nevertheless, by this way, well established linearization techniques, which lead to an efficient solution for $\Sigma_{c}$ cannot be applied.

\section{NUMERICAL EXAMPLE}

The considered system is the NN8 example, extracted from the publicly available benchmark collection $\mathrm{COMPl}_{e}$ ib [16], regarding only its first input and output, resulting thus in a SISO system. Its state space representation is given by

$$
\begin{aligned}
A_{p} & =\left[\begin{array}{rrr}
-0.25 & 0.1 & 1 \\
-0.05 & 0 & 0 \\
0 & 0 & -1
\end{array}\right], \quad B_{p}=\left[\begin{array}{l}
0 \\
0 \\
1
\end{array}\right], \\
C_{p} & =\left[\begin{array}{lll}
1 & 0 & 0
\end{array}\right], \quad D_{p}=0 .
\end{aligned}
$$

The exact design procedure is described in the next section.

\section{A. Design of controller $\Sigma_{c}$.}

As controller $\Sigma_{c}$ a Linear Quadratic Regulator is considered minimizing the cost function

$$
J=\int_{0}^{\infty} y^{2}(\tau)+0.01 u^{2}(\tau) d \tau,
$$

with a full state observer. The poles of the observer are placed in the positions [-2 -3-4], resulting in the controller 


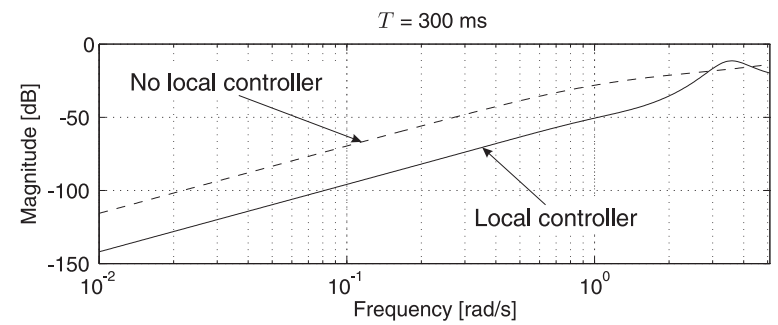

Fig. 2. Bode diagram of the sensitivity function with respect to time delay, of the systems with and without the transformation $M$, for $T=300 \mathrm{~ms}$.
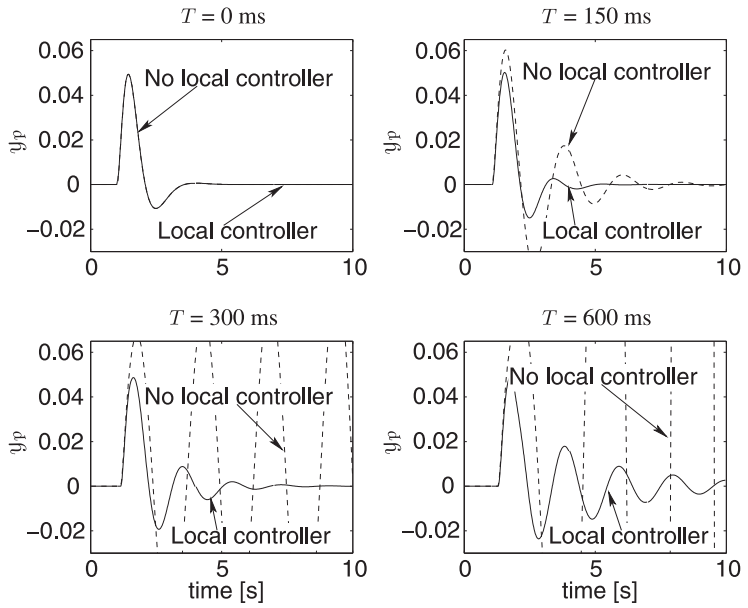

Fig. 3. Impulse response of the systems with and without the transformation $M$, for different values of the time delay.

$\begin{aligned} A_{c} & =\left[\begin{array}{rrr}-8 & 0.1 & 1 \\ -240 & 0 & 0 \\ -3.122 & -0.339 & -4.387\end{array}\right], B_{c}=\left[\begin{array}{r}-7.8 \\ -239.95 \\ 6\end{array}\right], \\ C_{c} & =[-9.122-0.339-3.387], \quad D_{c}=0 .\end{aligned}$

B. Design of transformation $M$

For the design of the transformation $M$, (9) is solved using the YALMIP Matlab toolbox [17] with the local solver PENBMI [18]. The gains $\gamma_{p}, \gamma_{c}$ are kept constant to $10^{6}$. The constraint $l_{1} \neq l_{4}$, which cannot be handled by the solver, is substituted by $l_{1}-l_{4} \geq 0.2$, in order to further avoid solutions close to singularity. The optimization problem is solved iteratively for decreasing values of $\gamma_{e x}$. A feasible solution was always obtained for all $\gamma_{e x} \geq 0.58$, independently of the initial values of $l_{1}, l_{2}, l_{3}, l_{4}$. The initial values only slightly affected the CPU optimization time, which for $\gamma_{e x}=0.58$ fluctuated from 3.6s to 4s, in an AMD Athlon 64 Dual Core Processor 3800+ CPU running under Debian Linux. The free parameter was fixed to $d=1$. The elements of the best obtained transformation are $a=22.51, b=151.083, c=$ $-0.017, d=1$ and the $H_{\infty}$ norm $\left\|G_{O L}\right\|_{\infty}=0.5767$.

\section{Simulations}

In the following, a comparison is presented between the systems with and without the transformation.
1) Sensitivity to time delay: The sensitivity function with respect to round trip time delay of the two systems, for round trip time delay $T=300 \mathrm{~ms}$, is shown in Fig. 2 . The sensitivity is plotted until the maximum cutoff frequency of the two closed loop systems. The sensitivity of the system with the transformation is less than the one without the transformation in almost all the frequencies of interest.

2) Impulse response: The impulse response to the plant input, is shown in Fig. 3 for $T=0,150,300,600 \mathrm{~ms}$. For zero time delay both systems give the same response. The system with the transformation remains stable in all cases, and its response is slightly affected by the time delay value. On the contrary, the system without the transformation is sensitive to time delay, and becomes unstable at $T=288 \mathrm{~ms}$.

\section{CONCLUSIONS}

In this article a novel control approach is proposed with a two degrees of freedom controller for delay-independent stability and low sensitivity to time delay. The first controller part guarantees specific performance measures for zero time delay, while the second part deals with delay-independent stability and low sensitivity to time delay. The necessary and sufficient conditions of the existence of a controller which guarantees delay-independent stability and a frequency dependent maximum of the norm of the sensitivity function with respect to time delay are formulated as a feasibility problem with polynomial matrix constraints. In simulations it is shown that the proposed formulation can give usefull solutions in non-trivial cases. In a numerical example the proposed approach significantly reduces the sensitivity to time delay. Future research is to approach multiple-inputmultiple-output systems, and time-varying delay.

\section{APPENDIX}

Firstly, we prove an alternative formulation of the static output feedback stabilizability problem for systems with $D \neq 0$. This is necessary as we cannot consider without loss of generality $D_{p}=D_{c}=0$ since the right and the left transformations $M$ and $M^{-1}$ are not independent systems.

Lemma 1: The closed loop system of a system $\Sigma$ (1) with a static output feedback gain matrix $K$, i.e.

$$
u=w+K y
$$

is strictly stable if and only if there is a symmetric positive definite matrix $P=P^{T}>0$ and a $\gamma \in \mathbb{R}^{+}$so that

$$
\begin{aligned}
& F=\left[\begin{array}{rr}
A^{T} P+P A & P B \\
B^{T} P & 0
\end{array}\right]+\left[\begin{array}{rr}
0 & I \\
C & D
\end{array}\right]^{T} Q\left[\begin{array}{rr}
0 & I \\
C & D
\end{array}\right] \leq 0 \\
& \text { with } Q=T^{T}\left[\begin{array}{rr}
I & 0 \\
0 & -\gamma^{2} I
\end{array}\right] T, \quad T=\left[\begin{array}{rr}
0 & I \\
I & -K
\end{array}\right] .
\end{aligned}
$$

Proof : Strict stability of LTI systems is equivalent to finite gain $L_{2}$ stability. Finite gain $L_{2}$ stability is equivalent to the existence of a quadratic positive definite function $V=x^{T} P x: \mathbb{R}^{n} \rightarrow \mathbb{R}$, i.e. a symmetric positive definite matrix $P=P^{T}>0$ so that for each $w \in \mathbb{R}^{m}$ the following holds

$$
\dot{V}+y^{T} y \leq \gamma^{2} w^{T} w \Rightarrow \dot{V}+\left[\begin{array}{c}
y \\
w
\end{array}\right]^{T}\left[\begin{array}{ll}
I & \\
0 & -\gamma^{2} I
\end{array}\right]\left[\begin{array}{c}
y \\
w
\end{array}\right] \leq 0 .
$$




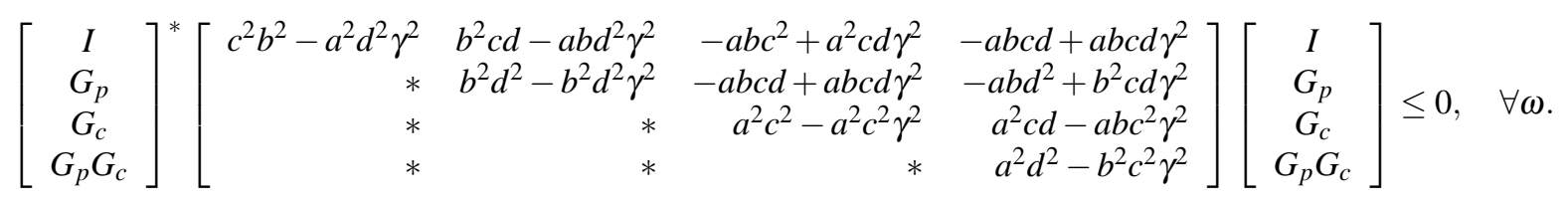

Equation (14) can be rewritten as

$$
\left[\begin{array}{c}
y \\
w
\end{array}\right]=\left[\begin{array}{rr}
0 & I \\
I & -K
\end{array}\right]\left[\begin{array}{l}
u \\
y
\end{array}\right] .
$$

Between the states of the plant $x$ and its input-output vector we have

$$
\left[\begin{array}{l}
u \\
y
\end{array}\right]=\left[\begin{array}{cc}
0 & I \\
C & D
\end{array}\right]\left[\begin{array}{l}
x \\
u
\end{array}\right]
$$

and further

$$
\dot{V}=2 x^{T} P[A x+B u]=\left[\begin{array}{l}
x \\
u
\end{array}\right]\left[\begin{array}{rr}
A^{T} P+P A & P B \\
B^{T} P & 0
\end{array}\right]\left[\begin{array}{l}
x \\
u
\end{array}\right] \text {. }
$$

Substituting (19)-(21) in (18) it results in

$$
\left[\begin{array}{l}
x \\
u
\end{array}\right]^{T} F\left[\begin{array}{l}
x \\
u
\end{array}\right] \leq 0
$$

Controllability implies that each state $x$ can be reached. Further, since the system is assumed to be well-posed, i.e. the matrix $[I-K D]$ is invertible, for the input signal $u$ we get $u=[I-K D]^{-1} w+[I-K D]^{-1} K C x$. Since $w$ can be freely chosen, so can $u$. Thus, necessary and sufficient condition for (22) is that the matrix F is negative semi-definite.

We are now able to state the proof of Theorem 2

Proof of Theorem 2: The fact that $\left\|G_{O L}\right\|_{\infty}$ is bounded by $\gamma_{e x}$ is equivalent to the strict stability of $\Sigma_{r}, \Sigma_{l}$ and

$$
G_{O L}(j \omega)^{*} G_{O L}(j \omega) \leq \gamma_{e x}^{2}, \quad \forall \omega .
$$

By substituting (6) in (23), and separating the terms with respect to $G_{p}, G_{c}, G_{p}^{*}, G_{c}^{*}$ and their products, (23) can be rewritten as in (17). By denoting the extended system (11) by $G_{e x}=\left[\begin{array}{lll}G_{p} & G_{c} & G_{p} G_{c}\end{array}\right]^{T}$, after some mathematical manipulation (17) becomes

$$
\begin{gathered}
{\left[\begin{array}{c}
I \\
G_{e x}(j \omega)
\end{array}\right]^{*} T_{e x}^{T}\left[\begin{array}{rr}
1 & 0 \\
0 & -\gamma_{e x}^{2}
\end{array}\right] T_{e x}\left[\begin{array}{c}
I \\
G_{e x}(j \omega)
\end{array}\right] \leq 0,} \\
\text { with } T_{e x}=\left[\begin{array}{cccc}
b c & b d & -a c & -a d \\
a d & b d & -a c & -b c
\end{array}\right] \\
\text { Setting } \quad b c=l_{1}, \quad b d=l_{2}, \quad a c=l_{3}, \quad a d=l_{4}
\end{gathered}
$$
under the constraint

$$
\frac{l_{1}}{l_{2}}=\frac{l_{3}}{l_{4}}=\frac{c}{d} \Rightarrow l_{1} l_{4}=l_{2} l_{3},
$$

the matrix $T_{e x}$ is given by (10). Using the KYP lemma, (24) is equivalent to the existence of a symmetric matrix $P_{e x}=P_{e x}^{T}$ so that $F_{e x} \leq 0$ (9).

The constraints $F_{p}, F_{c} \leq 0$ (9) imply the strict stability of the $\Sigma_{r}, \Sigma_{l}$. From (2) and (25) it is straightforward to see that $u_{p}=1 / a u_{r}-b / a y_{p}$, i.e. the feedback gain of $\Sigma_{p}$ in subsystem $\Sigma_{r}$ is $-b / a=-l_{1} / l_{3}$. Based on Lemma 1 strict stability of $\Sigma_{r}$ is equivalent to the existence of a symmetric positive definite matrix $P=P^{T}>0$ and a $\gamma \in \mathbb{R}^{+}$ so that $F \leq 0$ (15) with $T$ given by

$$
T=\left[\begin{array}{rr}
0 & 1 \\
1 & l_{1} / l_{3}
\end{array}\right] \text {. }
$$

By multiplying $F$ (15) with $l_{3}^{2}, F_{p} \leq 0$ (9) is obtained. Following the same procedure for $\Sigma_{l}$ and considering the negative feedback sign, $F_{c} \leq 0$ (9) is obtained. The last constraint $l_{1} \neq l_{4} \Rightarrow a d-b c \neq 0$ implies the invertibility of the mapping $M$.

For each $l_{1}, l_{2}, l_{3}, l_{4}$ under the restriction (26), one of the parameters $a, b, c, d$ can be freely chosen. We consider here $d$ as a free parameter, the other cases are equivalent. The rest of the parameters are given by $a=\frac{l_{4}}{d}, b=\frac{l_{2}}{d}, c=d \frac{l_{1}}{l_{2}}$.

\section{REFERENCES}

[1] Y. Tipsuwan and M. Y. Chow, "Control methodologies in network control systems," Control Engineering Practice, vol. 11, pp. 10991011, 2003.

[2] D. Hristu-Varsakelis and W. S. Levine, Handbook of Networked and Embedded Control Systems. Birkhäuser, 2005.

[3] J. P. Hespanha, P. Naghshtabrizi, and Y. Xu, "A survey of recent results in networked control systems," in Proceedings of the IEEE, vol. 95, no. 1, January 2007, pp. 138-162.

[4] K. Gu, V. Kharitonov, and J. Chen, Stability of Time-Delay Systems, B. Boston, Ed., 2003.

[5] J.-P. Richard, "Time-delay systems: an overview of some recent advances and open problems," Automatica, vol. 39, pp. 1667-1694, 2003.

[6] T. Matiakis and S. Hirche, "Memoryless input-output encoding for networked systems with unknown constant time delay," in Proceedings of 2005 IEEE Conference on Control Applications, October 2006, pp. $1707-1712$.

[7] T. Matiakis, S. Hirche, and M. Buss, "A novel input-output transformation method to stabilize networked control systems independent of delay," in Proceedings of 17th International Symposium Mathematical Theory of Networks and Systems, 2006, pp. 2891-2897.

[8] R. J. Anderson and M. W. Spong, "Bilateral control of teleoperators with time delay," IEEE Transactions on Automatic Control, vol. 34, no. 5, pp. 494-501, May 1989.

[9] G. Niemeyer and J. Slotine, "Stable adaptive teleoperation," International Journal of Oceanic Engineering, vol. 16, no. 1, pp. 152-162, 1991.

[10] C. Scherer and S. Weiland, Linear Matrix Inequalities in Control, E. U. of Technology, Ed., 2007. [Online]. Available: http://w3.ele.tue.nl/nl/mbs/cs/education/courses/disclmi

[11] R. Anderson and M. Spong, "Bilateral Control of Teleoperators with Time Delay," vol. 34, no. 5, pp. 494-501, 1989.

[12] R. Lozano, N. Chopra, and M. Spong, "Passivation of Force Reflecting Bilateral Teleoperators with Time Varying Delay," in Proceedings of the 8. Mechatronics Forum, Enschede, Netherlands, 2002, pp. 954962.

[13] B. Berestesky, N. Chopra, and M. W. Spong, "Discrete Time Passivity in Bilateral Teleoperation over the Internet," in Proceedings of the IEEE International Conference on Robotics and Automation ICRA'04, New Orleans, US, 2004, pp. 4557-4564.

[14] "A new reformulation-linearization technique for bilinear programming problems," Journal of Global Optimization, vol. 2, no. 4, pp. 379-410, December 1992.

[15] O. Toker and H. Ozbay, "On the NP-hardness of solving bilinear matrix inequalities andsimultaneous stabilization with static output feedback," in Proceedings of American Control Conference, ACC 1995., vol. 4, June 1995, pp. 2525-2526.

[16] F. Leibfritz, "Compleib: Constraint matrix-optimization problem library - a collection of test examples for nonlinear semidefinite programs, control system design and related problems," Technical Report, Department of Mathematics, University of Trier, 2004. [Online]. Available: http://www.complib.de

[17] J. Löfberg, "YALMIP : A toolbox for modeling and optimization in MATLAB," in Proceedings of the CACSD Conference, 2004. [Online]. Available: http://control.ee.ethz.ch/ joloef/yalmip.php

[18] M. Kočara and M. Stingl, "PENNON: a code for convex nonlinear and semidefinite programming," Optimization Methods and Software, vol. 18, 2003. 